\title{
Editorial: Hybrid Solutions for the Modeling of Complex Environmental Systems
}

\author{
Christian E. Vincenot ${ }^{1 *}$, Stefano Mazzoleni ${ }^{2}$ and Lael Parrott ${ }^{3}$ \\ ${ }^{1}$ Biosphere Informatics Laboratory, Department of Social Informatics, Kyoto University, Kyoto, Japan, ${ }^{2}$ Laboratory of Applied \\ Ecology and System Dynamics, Department of Agricultural Sciences, University of Naples Federico II, Portici, Italy, \\ ${ }^{3}$ Okanagan Institute for Biodiversity, Resilience, and Ecosystem Services, The University of British Columbia, Kelowna, BC, \\ Canada
}

Keywords: integration, multiscale, simulation, agent, system dynamics, machine learning, network, coupled

\section{The Editorial on the Research Topic}

\section{Hybrid Solutions for the Modeling of Complex Environmental Systems}

Hybrid modeling is the combination of simulatory approaches aimed at the accurate mechanistic modeling of complex dynamic systems (Parrott, 2011; Vincenot et al., 2011). It basically consists in the coupling-to a varying degree ranging from simple comparison to bridging to total fusion-of models based on different existing modeling techniques. This concept may therefore, in some regards, seem trivial and more relevant to engineering than scientific research. Yet

OPEN ACCESS

Edited by:

Marco Casazza,

Parthenope University of Naples, Italy

Reviewed by:

Laura Uusitalo,

University of Helsinki, Finland

*Correspondence: Christian E. Vincenot

christian@vincenot.biz

Specialty section: This article was submitted to Environmental Informatics, a section of the journal Frontiers in Environmental Science

Received: 29 May 2016 Accepted: 02 August 2016 Published: 29 August 2016

Citation:

Vincenot CE, Mazzoleni S and Parrott L (2016) Editorial: Hybrid Solutions for the Modeling of Complex Environmental Systems.

Front. Environ. Sci. 4:53. doi: 10.3389/fenvs.2016.00053 seeing this field of study through a purely technical eye produces a limited view of the complexity and open challenges relevant to the design and use of hybrid models. This shall be demonstrated throughout this book, in which we engage in showing the potential of hybrid modeling in environmental science in particular. The latter is a strongly interdisciplinary field devoted to the study of heterogeneous, highly dynamical, often non-linear, complex systems made of numerous different entities-rational or not-interacting in a changing environment.

Reasons underlying the use of hybrid models are actually multiple. Hybrid modeling, in the widespread meaning of hybrid system modeling, has been delineated in engineering as a purely technical solution to the challenge of representing concurrently discrete events and continuous processes, which are common in automated systems. This issue has been dominating also in environmental science, in which systems often exhibit the same constraints (see Vincenot et al., 2015 for a discussion of this aspect). However, hybrid modeling can have a range of motivations other than accurate time representation. In the study of ecological systems in particular, it actually stems from a philosophical root in its ability to merge reductionist and holistic views and thereby acknowledge the ontology of Nature (Vincenot et al., 2011). In microbiology, the organization of cells, which are discrete interacting entities of variable size, is dependent on exchanges of molecules, which are diffusing as continuous flows in space. Coupling both aspects requires a hybrid view of the system with both reductionist agents (i.e., cells) and aggregated flows (i.e., intercellular signaling processes) (Hay Mele et al.). In oceanography, Eulerian-Lagrangian approaches that couple aggregated biogeochemical models and physical spatial transport models are used to simulate in three dimensions tracer dynamics in moving fluids and thereby understand spatial patterns of plankton under oceanic currents (Chenillat et al.). On a different topic, Vincenot et al. (2016) similarly showed how the integration inside of a System Dynamics (SD) -Individual-based (IB) hybrid model of realistic plant-level processes 
(i.e., continuous metabolism as well as mechanistic seed dispersal events) on top of a basic hydrological submodel provides insights on the demographic response and spatial distribution of vegetation facing climatic stress. Using a comparable conceptual framework, Bradhurst et al. demonstrate that the simultaneous use of individual/agent-based and equationbased formulation of the pathogen transmission process at different levels leads to increased accuracy in epidemiological modeling.

Frequently, hybrid modeling arises naturally from the need to couple several existing models, each describing one part of a system in the most suitable manner with respect to modeling goals. Cross-scale ecological simulations are particularly prone to such settings. Girard et al. report on such a hybrid model featuring interactions between regionalscale climate, landscape-scale hydrology, and individual animals, each of them calling for the use of a dedicated modeling approach. The integration of economic or social dynamics in environmental models also typically leads to the same situation. For example, Drogoul et al. show here how they could improve the accuracy of governmental land-use change forecasts of the Mekong Delta in Vietnam by resorting to a hybrid model including social, ecological, and economic dimensions.

Hybrid modeling is often concurrently undertaken to reconciliate bottom-up and top-down views of a system. Ghersi summarizes the long-term work done at the Centre International de Recherche sur l'Environnement et le Développement (CIRED) on the coupling of bottom-up energy models with the IMACLIM-S macroeconomic model. Martin and Schlüter discuss a procedure to tackle the same methodological issue, which occurred in this case when linking the dynamics emerging from individual-level human decisions (micro-scale) and ecosystem-wide processes (macroscale) inside of a socio-ecological model supporting lake restoration.

Hybrid modeling is not solely a technical means to build large models through a combinatory approach. For the modeling of even a single component, the differences in characteristics or features exhibited by different paradigms give rise to hybrid concepts unlocking beneficial tradeoffs at runtime, between precision and performance for instance, by dynamically changing the representation of model components. This was termed dynamic model swapping (Vincenot et al., 2011) and discussed more specifically in the case of epidemiological modeling (Vincenot and Moriya, 2011) before being implemented by Gray and Wotherspoon (2012). The same authors detail here the unique methodological questions surfacing in such adaptive hybrid models, and

\section{REFERENCES}

Gray, R., and Wotherspoon, S. (2012). Increasing model efficiency by dynamically changing model representations. Environ. Model. Softw. 30, 115-122. doi: 10.1016/j.envsoft.2011.08.012 explain how a submodel selection strategy can be effectively performed by an intelligent monitor agent (Gray and Wotherspoon).

While hybrid models are not uncommon, this emerging discipline stands in need of further research establishing appropriate design and analysis methodologies. At the same time, there is still a clear necessity to explore new modeling avenues for environmental science. Advancements in the field of engineering especially can provide new simulative approaches and opportunities for model coupling to support a better rendering of complex environmental systems. Among others, Generalized Hybrid Petri Nets (GHPN) offer a framework to help formalize and visualize processes. The value of this approach is illustrated here in a study of Chagas disease transmission (Herajy and Heiner). In a different manner, machine learning, which is being increasingly applied across scientific disciplines, can be integrated in deterministic models to include data-driven components (Goldstein and Coco). Bayesian approaches can also serve to infer model structure and support the parameterization of individual-based models through data integration (Parise et al.). Agents can thereby be enhanced with human decision-making capabilities to study socio-ecological issues (Pope and Gimblett; an applied study on the effects of riparian corridors in the Sonoran desert).

The non-linear, self-organized dynamics of complex systems, including environmental ones, makes their behavioral trends extremely difficult to predict. Hybrid models, which typically represent the dynamics of a system over a wide parameter space, can be used to explore the envelope of possible, probable and plausible system trajectories. This is exemplified in the work presented by Tareen et al., who show how the simplification of the relationships between interacting components in terms of feedbacks in the frame of a parametric linear hybrid automaton allows for the isolation and study of particular system trajectories.

Through developments such as the ones presented in this book, hybrid modeling is expected to ultimately shift the fundamental question of environmental modeling from the usual "With this technique, how can this system be represented?" to "Which combination of paradigms would best render this set of processes?" Doing so will bring the discipline closer to understanding and successfully reproducing the complex dynamics of environmental systems through models.

\section{AUTHOR CONTRIBUTIONS}

All authors listed, have made substantial, direct and intellectual contribution to the work, and approved it for publication. 
a system dynamics—individual-based hybrid model. Front. Plant Sci. 7:636. doi: 10.3389/fpls.2016.00636

Vincenot, C. E., Giannino, F., Rietkerk, M., Moriya, K., and Mazzoleni, S. (2011). Theoretical considerations on the combined use of System Dynamics and individual-based modeling in ecology. Ecol. Modell. 222, 210-218. doi: 10.1016/j.ecolmodel.2010. 09.029

Vincenot, C. E., Mazzoleni, S., Moriya, K., Cartenì, F., and Giannino, F. (2015). How spatial resource distribution and memory impact foraging success: a hybrid model and mechanistic index. Ecol. Complex. 22, 139-151. doi: 10.1016/j.ecocom.2015.03.004

Vincenot, C. E., and Moriya, K. (2011). Impact of the topology of metapopulations on the resurgence of epidemics rendered by a new multiscale hybrid modeling approach. Ecol. Inform. 6, 177-186. doi: 10.1016/j.ecoinf.2011. 04.002

Conflict of Interest Statement: The authors declare that the research was conducted in the absence of any commercial or financial relationships that could be construed as a potential conflict of interest.

Copyright (c) 2016 Vincenot, Mazzoleni and Parrott. This is an open-access article distributed under the terms of the Creative Commons Attribution License (CC BY). The use, distribution or reproduction in other forums is permitted, provided the original author(s) or licensor are credited and that the original publication in this journal is cited, in accordance with accepted academic practice. No use, distribution or reproduction is permitted which does not comply with these terms. 